\title{
Parton shower effects in central scalar production at the LHC
}

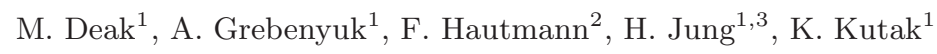 \\ 1- Deutsches Elektronen Synchrotron, Hamburg \\ 2- Theoretical Physics Department, University of Oxford \\ 3- Physics Department, University Antwerp
}

\begin{abstract}
We use Monte Carlo simulations of scalar boson production at the LHC to investigate the role of transverse momentum dependent parton showers and comment on their possible impact on modeling of multi-parton interactions.
\end{abstract}

\section{Introduction}

The treatment of parton showers and the treatment of the recoils in the parton shower is essential for a proper description of the $p_{t}$ spectrum in Drell Yan processes at $p p$ collisions [1] and has influence on the amount of multi-parton interactions needed to describe the measurements. Even more, parton showers which are not ordered in transverse momentum could also considerably contribute to what is typically associated with the underlying event. Details of the final state are best studied and understood using unintegrated parton distributions $[2-6]$.

To study the effect of such parton showers and its contribution to the underlying event, we investigate a gluon induced process which produces a color singlet scalar system in the final state, here $g g \rightarrow h^{0}$.

\section{Monte Carlo models}

The Monte Carlo generators we will use in this study are Pythia [7] and Cascade [8]. In PYTHIA we use a parameter set allowing for more initial and final state radiation (Perugia tune hard) [1] with the $k_{t}$-ordered parton shower. CASCADE [9] uses the unintegrated parton distribution functions convoluted with off - mass shell matrix elements for the hard scattering [10-14]. A general discussion on unintegrated gluon densities can be found in $[19,20]$. Here we use the CCFM $[15,16]$ unintegrated gluon densities [17]. ${ }^{\mathrm{a}}$

An important feature of using unintegrated parton density functions is that the kinematics of the whole event are fixed from the beginning [2], in contrast to the collinear approach where a mapping of the $n+1$ to the $n$-partonic configuration has to be performed with the associated rearrangement of kinematics [21].

\section{$3 \quad$ Parton showering studies}

For our study we classify the final state in hadronic collisions into 4 different areas: $a$. hard process and hard radiation, $b$. hard process and semi-hard radiation, $c$. hard process and

\footnotetext{
${ }^{a}$ We observe that the unintegrated formulation may provide a natural framework to incorporate high parton density effects as well, see e.g. [18].
} 
soft radiation and $d$. soft process and soft radiation.

\subsection{Hard process and hard radiation}

The treatment of hard radiation in the high energy limit is given in $[11,13]$. As discussed in [20] the hard process described by offshell matrix elements covers a significant part from next-to-leading order corrections in collinear factorisation. This feature is often used to estimate the size of higher order corrections in collinear factorisation (see for example [22]). A comparison of the prediction from CASCADE with NLO prediction for dijets in DIS as well as for top production at LHC is given in [19]. The energy and angular distributions of the quarks in $\gamma^{*} g^{*} \rightarrow q \bar{q}$ or $g^{*} g^{*} \rightarrow Q \bar{Q}$ agree pretty well with those obtained from NLO calculations. As shown in [19] also the transverse momentum of the $q \bar{q}$ pair agrees well both in DIS and $p p$ with the NLO calculation. Thus, the first hard

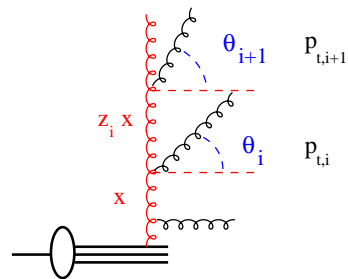

Figure 1: Initial parton cascade with angular ordering parton radiation is also reasonably well described with $k_{\perp}$-factorisation and the CCFM uPDFs. Both the off-shell matrix elements and the unintegrated gluon density are needed for obtaining this agreement.

Whereas a NLO calculation in $p p \rightarrow Q \bar{Q}+X$ would allow only for one additional hard parton radiation, the off-shell matrix element $g^{*} g^{*} \rightarrow Q \bar{Q}$ allows for any number of hard parton emissions, while the gluon transverse momentum is restricted by kinematics and the angular ordering constraint.

Semi-hard radiation is essentially treated by the parton shower. The evolution of the CCFM parton densities is performed with a Monte Carlo method allowing the full and consistent treatment of the kinematics and the real emissions already during the evolution (as shown in [8]). In CASCADE the parton shower is treated in a backward approach for efficiency reasons, but reproduces exactly the distributions of emitted partons from the evolution. The CCFM formulation given in [16] is an explicit shower equation, which in the limit of $q_{i}>q_{i-1}$ reproduces the DGLAP equation.

The angular ordering condition in CCFM (see fig. 1) is given by $q_{i}>z_{i-1} q_{i-1}$ with $q_{i}=\frac{p_{t i}}{1-z_{i}}$ being the rescaled transverse momenta of the emitted partons and $z_{i}=\frac{x_{i}}{x_{i-1}}$ being the momentum fraction. The angular ordering condition allows for transverse momenta larger than the one of the hard $2 \rightarrow 2$ process. In contrast the large $x$ angular ordering condition used in HERWIG $q_{i}>q_{i-1}[23]$ (which reduces to $p_{t}$ ordering for not too large $z$ ) implies that the hard scattering has the largest transverse momentum.

\subsection{Hard process and semi-hard radiation}

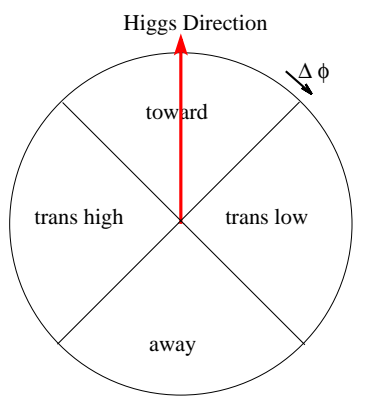

Figure 2: Different regions in $\phi$ with respect to the Higgs directions

In [19] a detailed comparison of the measured azimuthal difference in DIS di- and three jet events with the prediction from CASCADE is performed. The region of $\Delta \phi \sim 180^{\circ}$ is where soft gluon resummation can be applied and the region of small $\Delta \phi \sim 30^{\circ}$ is driven by hard parton radiation, thus offering a significant test of the quality of hard to semi-hard parton showers over the full region of phase space. The 
description of the measurement is good, giving confidence on the applicability of the method also for other processes. Approaches based on parton showers in collinear factorisation (HERWIG) cannot describe the shape of the $\Delta \phi$ distribution.

Next we study radiation associated with standard model Higgs boson production. The direction of the Higgs boson in the azimuthal plane defines the origin of the system, as shown in fig. 2. Four regions in the azimuthal plane are defined (following the CDF analysis of the underlying event [24]). In fig. 3 we show the average multiplicity for mini-jets with $E_{t}>15$ $\mathrm{GeV}$ and with $E_{t}>5 \mathrm{GeV}$ at LHC energies $(\sqrt{s}=14 \mathrm{TeV})$ in the 4 different regions of $\phi$ as a function of the Higgs transverse momentum.
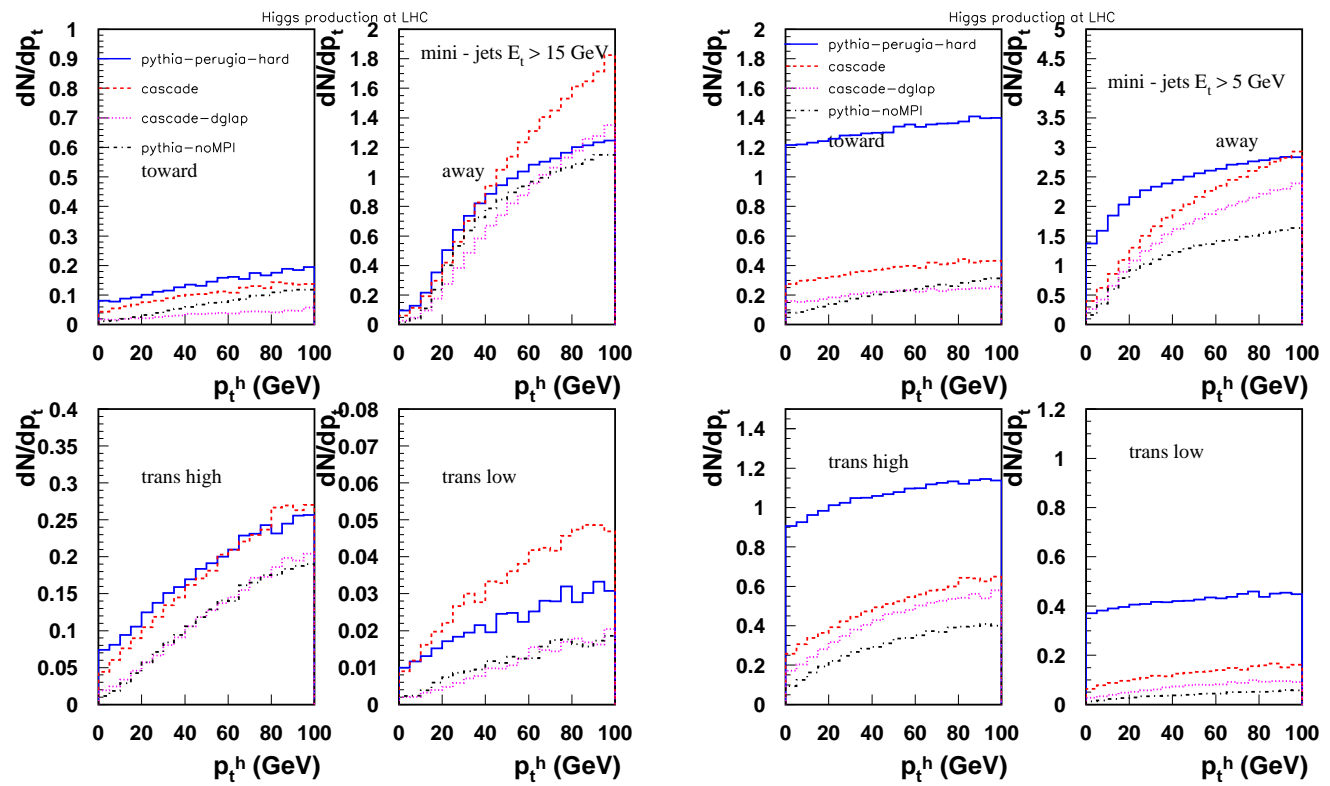

Figure 3: Multiplicity of jets as a function of the transverse momentum of the Higgs in different regions of $\phi$ as predicted from Cascade and from Pythia. Shown is also the prediction using CASCADE in the DGLAP mode. The left figure shows the prediction for mini-jets with $E_{T}>15 \mathrm{GeV}$, the right figure for $E_{T}>5 \mathrm{GeV}$.

The prediction of CASCADE is compared with predictions from PyTHIA [1]. For comparison, CASCADE is also run in the DGLAP mode (cascade-dglap), with the off-shell matrix element [6] replaced by the on-shell approximation and the parton showers are evolved with the one-loop splitting function and an upper restriction on the transverse momentum $p_{t}<\sqrt{m_{h}^{2}+p_{t h}^{2}}$. For mini-jets with $E_{T}>15 \mathrm{GeV}$ CASCADE in the DGLAP mode reproduces nicely the prediction of PYTHIA without multiparton interactions in both transverse regions. CASCADE with CCFM evolution and off-shell matrix element predicts higher activities in the transverse as well as in the toward regions, and is close or larger than the prediction of PYтнIA including multiparton interactions. In the away region the slope is steeper than predicted from PyтнIA. Lowering the transverse momentum cut of the minijets to $E_{T}>5 \mathrm{GeV}$, CASCADE still predicts a larger multiplicity than PythiA without 
multiparton interactions, but falls clearly below the prediction including multiparton interactions. This illustrates the onset of hard perturbative contributions from the parton showers, which are simulated in PYTHIA with multiparton interactions.

In fig. 4 we show the transverse momentum spectrum of the Higgs boson obtained from CASCADE with CCFM evolution and in DGLAP mode with the on-shell matrix element. Also shown is the prediction from PYTHIA and the resummed NLO calculation [25]. CASCADE with CCFM evolution agrees best with the resummed calculation in the region $p_{t h}<60 \mathrm{GeV}$. It is important to note, that the parton showers in CASCADE are determined by the uPDF, which is fixed from fits to inclusive cross section measurements [17]: once these parameters are determined, there is no freedom left to

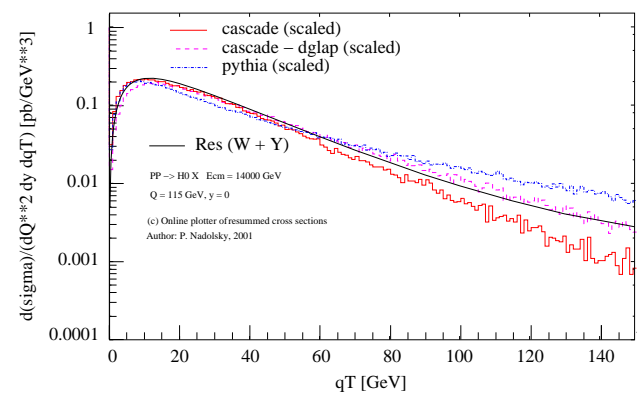

Figure 4: Transverse momentum spectrum of the Higgs boson change parameters on the initial state parton shower.

\subsection{Hard process and soft radiation}

The phase space region where soft radiation plays a significant role is the region of minimal transverse energy in the $\phi$ plane. This is the region where multiparton interactions should be visible. We have also studied the multiplicity of charged particles (with $p_{t}>150 \mathrm{MeV}$ ) in the process $g g \rightarrow h^{0}$. The prediction from PYтнIA including multiparton interactions is clearly above the prediction from CASCADE, however the multiplicity predicted from CASCADE is significantly larger than the one predicted by PYTHIA without multiparton interactions (not shown). The prediction using CCFM evolution shows significantly more activity in all regions. Thus even for the soft contribution in the charged particle multiplicity the treatment of parton showers is important.

\subsection{Soft process and soft radiation}

The region of soft processes and soft radiation is the region of minimum bias events where elastic and soft diffractive processes play a role. This is not (yet) considered in CASCADE.

\section{Conclusion}

We have studied the contribution of parton showers to observables sensitive to multiparton interactions. We use scalar production in $p p$ collisions as an example for a gluon-dominated process.

Parton showers based on the CCFM evolution equation convoluted with the off-shell matrix elements give a significantly larger multiplicity of charged particles and mini-jets compared to what is expected from the conventional DGLAP based parton shower approach including multiparton interactions. The benchmarks for parton shower are jet cross sections in DIS obtained from HERA, as they are very little influenced by multi-parton interactions. It could be shown, that the CASCADE Monte Carlo event generator not only successfully 
describes DIS and photoproduction cross section, but also is applicable to processes at the LHC. The prediction of mini-jet multiplicities in the transverse region is higher than what is predicted from a PYTHIA simulation including multiparton interactions.

\section{Acknowledgements}

Many thanks go to G. Hesketh, A. Knutsson and L. Motyka for making the session on hadronic final states at DIS09 so interesting and challenging. It is a pleasure to thank C. Glasman, C. Uribe-Estrada, A. Sabio Vera and especially J. Terron for the perfect organisation of this very interesting and fruitful workshop.

\section{References}

[1] P. Z. Skands (2009). arXiv:0905.3418.

[2] J. Collins and H. Jung (2005). hep-ph/0508280.

[3] L. Lonnblad. Prepared for 12th International Workshop on Deep Inelastic Scattering (DIS 2004), Strbske Pleso, Slovakia, 14-18 Apr 2004.

[4] L. Lonnblad and M. Sjodahl, JHEP 02, 042 (2004). hep-ph/0311252.

[5] H. Jung, Mod. Phys. Lett. A19, 1 (2004). hep-ph/0311249.

[6] F. Hautmann, Phys. Lett. B535, 159 (2002). hep-ph/0203140.

[7] T. Sjostrand, S. Mrenna, and P. Skands, JHEP 05, 026 (2006). hep-ph/0603175.

[8] H. Jung and G. P. Salam, Eur. Phys. J. C19, 351 (2001). hep-ph/0012143;

H. Jung, Comput. Phys. Commun. 143, 100 (2002). hep-ph/0109102;

H. Jung, The CASCADE Monte Carlo. http://www.desy.de/ jung/cascade, 2009.

[9] Slides:

http://indico.cern.ch/materialDisplay.py?contribId=278\&sessionId=3\&materialId=slides\&confId $=53294$.

[10] S. Catani, M. Ciafaloni, and F. Hautmann, Nucl. Phys. B366, 135 (1991).

[11] S. Catani, M. Ciafaloni, and F. Hautmann, Phys. Lett. B242, 97 (1990).

[12] S. Catani, M. Ciafaloni, and F. Hautmann, Phys. Lett. B307, 147 (1993).

[13] J. C. Collins and R. K. Ellis, Nucl. Phys. B360, 3 (1991).

[14] E. M. Levin, M. G. Ryskin, Y. M. Shabelski, and A. G. Shuvaev, Sov. J. Nucl. Phys. 53, 657 (1991).

[15] M. Ciafaloni, Nucl. Phys. B296, 49 (1988);

S. Catani, F. Fiorani, and G. Marchesini, Phys. Lett. B234, 339 (1990);

S. Catani, F. Fiorani, and G. Marchesini, Nucl. Phys. B336, 18 (1990).

[16] G. Marchesini, Nucl. Phys. B445, 49 (1995). hep-ph/9412327.

[17] M. Hansson and H. Jung (2007). 0707.4276;

H. Jung, A. V. Kotikov, A. V. Lipatov, and N. P. Zotov (2006). hep-ph/0611093.

[18] E. Avsar and E. Iancu, Phys. Lett. B673, 24 (2009). 0901.2873;

E. Avsar and E. Iancu (2009). 0906.2683;

K. Kutak and H. Jung (2008). 0812.4082.

[19] F. Hautmann and H. Jung, JHEP 10, 113 (2008). 0805.1049.

[20] Small x Collaboration, J. R. Andersen et al., Eur. Phys. J. C48, 53 (2006). hep-ph/0604189; Small x Collaboration, J. R. Andersen et al., Eur. Phys. J. C35, 67 (2004). hep-ph/0312333; Small x Collaboration, B. Andersson et al., Eur. Phys. J. C25, 77 (2002). hep-ph/0204115.

[21] S. Frixione, P. Nason, and B. R. Webber, JHEP 08, 007 (2003). hep-ph/0305252.

[22] S. Marzani, R. D. Ball, V. Del Duca, S. Forte, and A. Vicini, Nucl. Phys. B800, 127 (2008). 0801.2544.

[23] G. Marchesini and B. R. Webber, Nucl. Phys. B310, 461 (1988).

[24] CDF Collaboration, A. A. Affolder et al., Phys. Rev. D65, 092002 (2002).

[25] F. Landry, R. Brock, P. M. Nadolsky, and C. P. Yuan, Phys. Rev. D67, 073016 (2003). hep-ph/0212159.

DIS 2009 